\title{
The $\mathbf{C} 7$ pedicle as a superior fixation point in spinal stabilization for spinal metastatic disease
}

\author{
Harjot Thind ${ }^{1,2}$, Andrew J. Fabiano ${ }^{1,2}$ \\ ${ }^{1}$ Department of Neurosurgery, Roswell Park Cancer Institute, Buffalo, New York, USA; ${ }^{2}$ Department of Neurosurgery, Jacobs School of Medicine \\ and Biomedical Sciences, University at Buffalo, State University of New York, Buffalo, New York, USA \\ Correspondence to: Andrew J. Fabiano, MD. Department of Neurosurgery, Roswell Park Cancer Institute, Elm \& Carlton Streets, Buffalo, NY 14263, \\ USA. Email: Andrew.Fabiano@Roswellpark.org.
}

\begin{abstract}
Spinal metastatic disease (SMD) often requires spinal stabilization; however, the cervicothoracic junction can be a challenging area to instrument. An anterior approach may require division of the sternum. A posterior or posterolateral approach may rely on cervical lateral mass screws for superior construct fixation that are more prone to pullout than screws placed in a pedicle. The C7 pedicle is able to support pedicle screw fixation in most instances based on morphological features of the vertebra. When the C7 pedicle is used as a superior fixation point, it aligns with the thoracic pedicles below to create a streamlined posterior construct. In this study, patients undergoing posterior stabilization with $\mathrm{C} 7$ pedicle superior fixation were examined. One hundred and thirty-nine consecutive spinal operations at a National Cancer Institute designated cancer center were retrospectively reviewed to identify patients who underwent spinal stabilization for SMD with a C7 pedicle screw placed as the superior fixation point of a posterior construct. Patient age, the primary disease, and clinical and radiographic information were identified. Follow-up duration was noted, and follow-up outcomes were recorded on the basis of the clinical history and the findings on computed tomography (CT) spinal imaging. Three patients were identified who underwent separation surgery for SMD that included posterior spinal stabilization with $\mathrm{C} 7$ pedicle screws as the superior fixation point. The average patient age was 70 years and one patient was a woman. The average follow-up time was 20.7 months. There were no occurrences of hardware failure, neurologic deterioration, or protracted pain in the cases analyzed. Overall, there were good surgical outcomes with improvement in pain without neurovascular injury or evidence of hardware failure during follow-up evaluation. These findings add to a small but notable number of studies showing the effectiveness of $\mathrm{C} 7$ pedicle screws as a superior fixation point in spinal oncology, specifically in metastatic lesions. In our experience the C7 pedicle has provided a useful superior fixation point solution for the posterior stabilization of high thoracic vertebral body metastases. This surgical option may help spinal surgeons address the stabilization of SMD in the cervicothoracic region.
\end{abstract}

Keywords: Cervical vertebrae; neoplasm metastasis; pedicle screws; spine; spinal stabilization

Submitted Sep 28, 2017. Accepted for publication Dec 07, 2017.

doi: $10.21037 /$ jss.2018.03.07

View this article at: http://dx.doi.org/10.21037/jss.2018.03.07

\section{Introduction}

Spinal stabilization is an integral part of the management of patients with spinal metastatic disease (SMD). Effective spinal stabilization in patients with SMD can preserve neurologic function, prevent spinal deformity, reduce pain and the need for opioid medications, and prolong the patient's survival (1). Spinal instrumentation is often placed as part of a separation surgery for SMD with the goals of decompressing the neural elements and stabilizing the affected area of the spinal column $(1,2)$.

The cervicothoracic junction can be a challenging area 

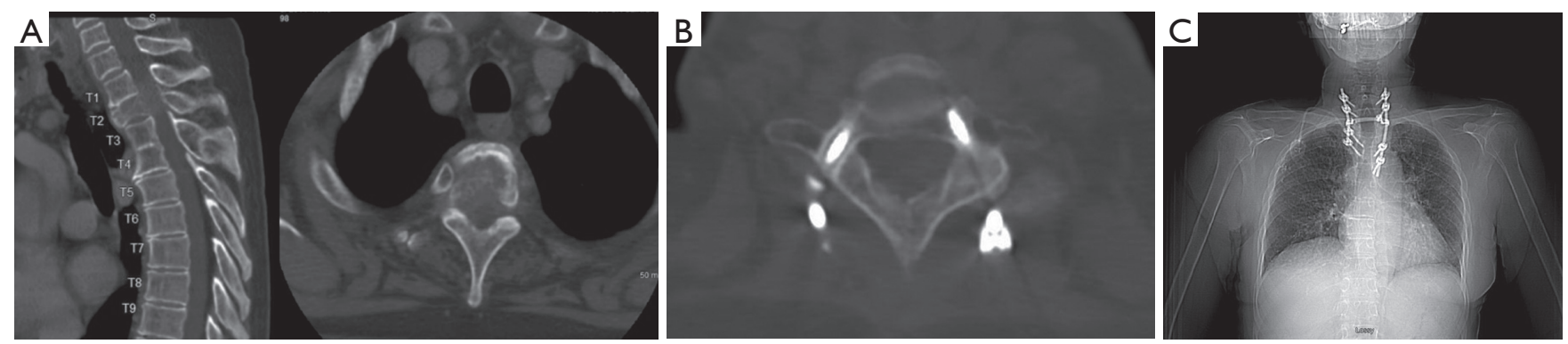

Figure 1 Case 1. (A) Preoperative CT scan (left) and axial image at level of T2 (right) showing pathologic facture; (B) T2 axial view of C7 pedicle screws on 32-month postoperative CT scan; (C) anteroposterior radiograph of C7-T5 posterior pedicle screw instrumentation construct at 32 months postoperatively.

to stabilize. This spinal region is a transition zone from a lordotic and mobile cervical spine to a kyphotic and rigid thoracic spine. High thoracic vertebral body lesions, such as T2 vertebral body metastases, may be particularly difficult to stabilize with instrumentation. An anterior approach to the tumor may require division of the sternum and retraction of the mediastinum. A posterior or posterolateral approach may rely on cervical lateral mass screws for superior construct fixation. These screws are more prone to pullout than screws placed in a pedicle $(3,4)$.

The C7 pedicle is able to support pedicle screw fixation in most instances based on its morphological features $(5,6)$. However, in posterior cervicothoracic constructs, the C7 level is often omitted due to its anatomical relationship to fixation points located above and below the $\mathrm{C} 7$ pedicle. Yet, when the $\mathrm{C} 7$ pedicle is used as a superior fixation point, it aligns with the thoracic pedicles below to create a streamlined posterior construct. In our experience, the C7 pedicle has provided a useful solution as a superior fixation point for the posterior stabilization of high thoracic vertebral body metastases. This surgical option may help spinal surgeons address SMD in the cervicothoracic region.

In this study, 139 consecutive spinal operations performed from 2012 through 2016 at a National Cancer Institute designated cancer center were retrospectively reviewed. Patients who underwent spinal stabilization for SMD with a C7 pedicle screw as the superior fixation point in a posterior spinal instrumentation construct were identified. Patients in whom anterior instrumentation was also placed (i.e., corpectomy cage) were excluded. The patient's age, primary disease, and clinical and radiographic information were identified. Operative details and the type of intraoperative imaging used for guidance during screw placement were assessed. The follow-up duration was noted, and follow-up outcomes were recorded on the basis of clinical history and computed tomography (CT) spinal imaging.

\section{Case presentation}

We identified three patients who underwent separation surgery for SMD that included posterior spinal stabilization instrumentation with $\mathrm{C} 7$ pedicle screw placement. In all three cases, the $\mathrm{C} 7$ pedicle screw was the superior fixation point. The average patient age was 70 years. One patient was a woman. The primary diagnoses were breast adenocarcinoma, liposarcoma of the lower extremity, and thyroid carcinoma, respectively. The average length of follow-up was 20.7 months (32, 24, and 6 months, respectively). There were no occurrences of hardware failure, neurologic deterioration, or protracted pain in the three cases analyzed.

\section{Case 1}

A 70-year-old woman with a history of breast adenocarcinoma presented with right upper extremity and intrascapular pain and myelopathy. Imaging revealed a T2 pathologic compression fracture with significant spinal cord compression (Figure 1A). The patient's primary disease prognosis was $>6$ months. The patient underwent T2 laminectomy and epidural tumor removal with C7-T5 posterior pedicle screw instrumentation (Figure 1B). Instrumentation was unilaterally extended to T5 because the T3 pedicle on the left side was infiltrated with tumor and was not an adequate fixation point. Conventional 2-dimensional fluoroscopy was used intraoperatively to place the instrumentation. Postoperatively, the patient 

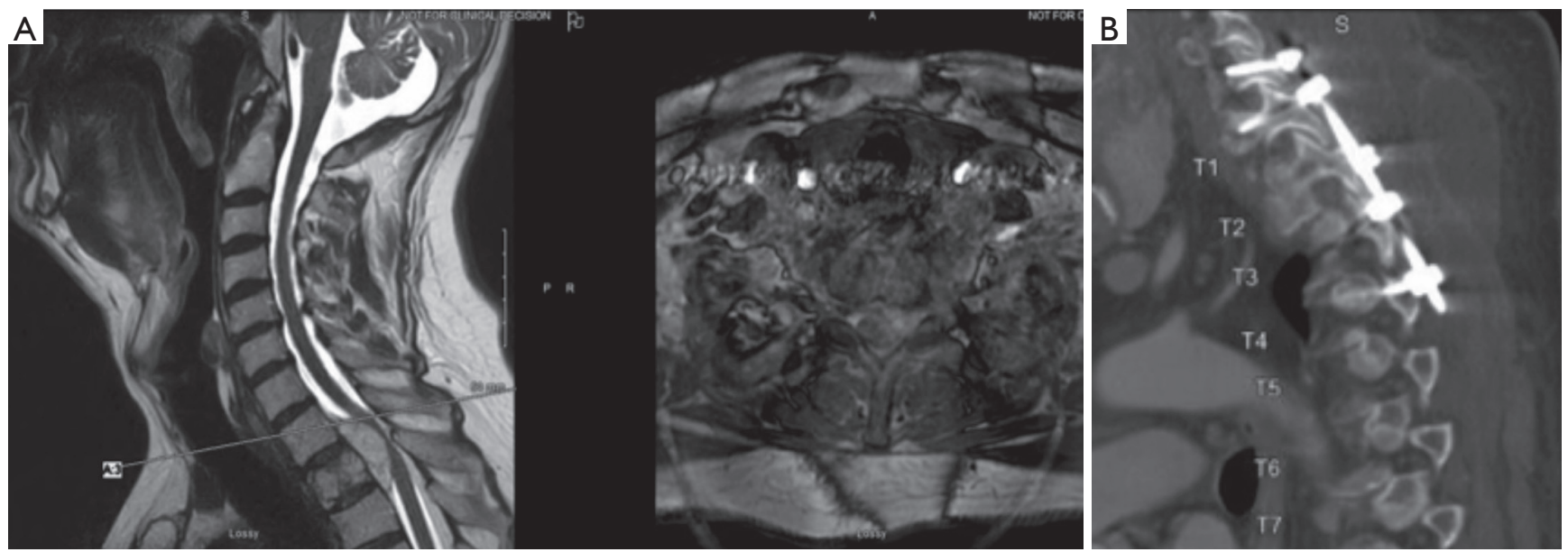

Figure 2 Case 2. (A) Preoperative MR sagittal image showing T2 pathologic fracture with epidural disease (left) and axial image (right) at level of T1 as indicated by scout line; (B) postoperative CT scan of C7 to T4 posterior pedicle screw instrumentation.

underwent fractionated radiotherapy from $\mathrm{C} 7$ to $\mathrm{T} 4$ with a dose of 30 Gy in 10 fractions. At follow-up at 32 months, the patient's pain had resolved; she was ambulating well without an assistive device; and follow-up radiography (Figure $1 C$ ) and CT imaging showed a stable spinal alignment.

\section{Case 2}

A 64-year-old man with a history of myxoid liposarcoma of his left lower extremity presented with intrascapular pain and rapid neurologic deterioration. The patient exhibited decreased sensation from the nipples downward and progressive bilateral lower-extremity weakness leading to a nonambulatory status. On neurologic examination, neither lower extremity was antigravity; there was decreased sensation to pinprick below T3; and the lower extremities were hyperreflexia with clonus. Imaging revealed a $\mathrm{T} 2$ pathologic compression fracture with a sizable epidural component causing spinal cord compression (Figure 2A). The patient's overall prognosis was $>6$ months. Therefore, on the day of consultation, the patient underwent emergent decompressive T1-3 laminectomies and epidural tumor removal. On postoperative day 6 , he underwent a planned staged surgery for the placement of C7-T4 posterior pedicle screw instrumentation (Figure 2B). Conventional 2-dimensional fluoroscopy was used intraoperatively to place the instrumentation. The patient underwent fractionated postoperative radiotherapy to $\mathrm{T} 2$. The patient recovered neurologic function and was able to ambulate with a cane. He had no cervical or thoracic pain. Followup spinal CT imaging at 24 months showed a stable spinal alignment.

\section{Case 3}

A 75-year-old man with a history of prostate carcinoma, thyroid carcinoma, and basal cell skin cancer presented with right scapular, shoulder, and right upper extremity pain along with paresthesia in that arm. On examination, he had right hand grip weakness with decreased sensation in a C8 distribution on the right and lower extremity myelopathy. Imaging revealed a $\mathrm{T} 1$ bony lesion with an epidural extension causing marked spinal cord compression (Figure 3A). The tumor also extended anteriorly in the cavity of the previously resected thyroid carcinoma. Preoperative biopsy of the mass determined that it was thyroid carcinoma. A head and neck oncologic surgeon was consulted. The surgeon cautioned against an anterior approach given the previous neck surgery and the current location of disease. The patient's overall prognosis was $>6$ months. $\mathrm{He}$ underwent $\mathrm{C} 7-\mathrm{T} 3$ posterior pedicle screw instrumentation with $\mathrm{T} 1$ right hemilaminectomy and epidural tumor resection, partial C7 laminectomy, and C8-T1 right-sided foraminotomies (Figure 3B). Intraoperative 3-dimensional imaging and spinal neuronavigation were used to place the instrumentation. Postoperatively, the patient underwent stereotactic radiosurgery to the $\mathrm{T} 2$ tumor (800 cGy delivered in 1 fraction to C7-T2). At 6 months postoperatively, the patient's pain was controlled; he did not 

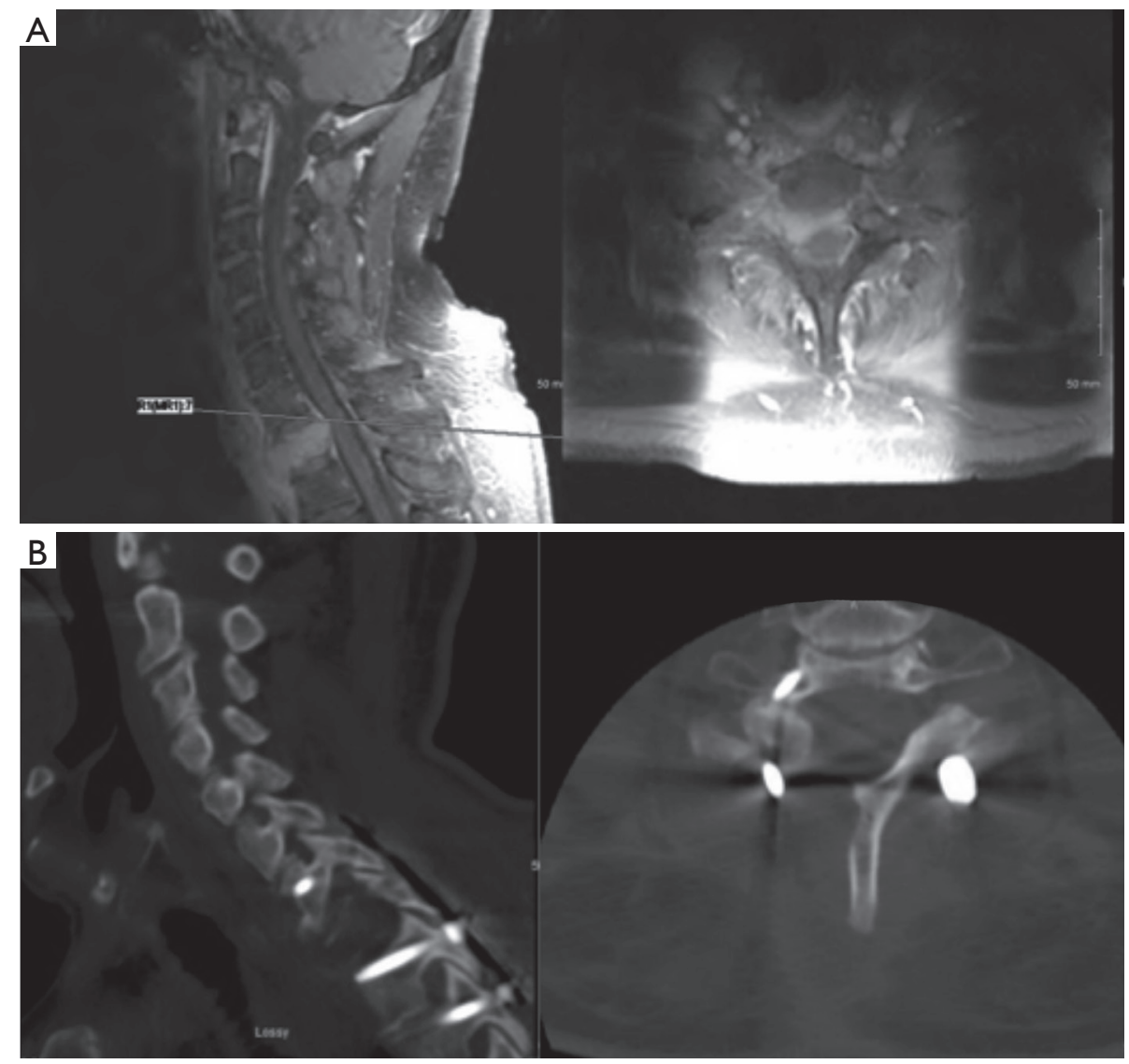

Figure 3 Case 3. (A) Preoperative sagittal MR image showing T1 bony lesion with an epidural extension (left). Axial imaging at T1 level as indicated (right). (B) Postoperative CT scan of C7-T3 posterior pedicle screw instrumentation with T1 right hemilaminectomy and epidural tumor resection, partial C7 laminectomy, and C8-T1 right-sided foraminotomies (left). Axial imaging at C7 level (right) showing placement of the $\mathrm{C} 7$ pedicle screw.

require medication and he ambulated without assistance. Spinal CT imaging showed a stable spinal alignment.

\section{Discussion}

We described our experience using the $\mathrm{C} 7$ pedicle screw as a superior fixation point in SMD patients who had high thoracic vertebral body lesions. Overall, we have had good surgical outcomes. All patients improved in pain, and those presenting with weakness also improved. Patients did not experience notable postoperative complications or need for further operations. Neurovascular injuries (intraor postoperatively) did not occur. All of the patients had good placement of C7 pedicle screws with no evidence of hardware failure postoperatively. Our average follow-up time of 20.7 months is adequate considering the overall prognosis of patients with SMD (7-9).
High thoracic vertebral body lesions present a difficult surgical stabilization problem due to the natural anterior biomechanical vector of the cervical spine relative to the thoracic spine. This is further complicated by the small anatomical size of cervical pedicles, which obviates screw placement, and the low pullout resistance of cervical lateral mass screws relative to thoracic or lumbar pedicle screws $(3,4)$. An anterior approach in this location often requires division of the sternum, which increases surgical morbidity (10). Individual clinical circumstances may also preclude an anterior approach. For example, our patient in Case 3 had already undergone an anterior neck dissection for a thyroid carcinoma. Therefore, a posterior approach was preferred.

Previous reports have described the use of cervical pedicle screws in spinal tumors. Placantonakis et al. and Oda et al. report a $0 \%$ hardware failure due to pullout of screws $(11,12)$. Mazel et al. report that C7 pedicle screws in spinal 
tumors of the cervicothoracic junction had no evidence of hardware failure (13). Pedicles provide stronger fixation than lateral mass or laminar screws at the C7 level (14). Rath et al. report no hardware failure or need for further surgery in a variety of pathologic conditions requiring cervicothoracic pedicle screw placement (15). Their results were not limited to neoplastic lesions.

However, some older studies of cervicothoracic junction spine surgery for neoplastic lesions did not consider or advocated against cervical, or specifically C7, pedicle screws due to risk of neurovascular injury $(16,17)$. Although it has been shown that there is an increased risk of damage to neurovascular structures from cervical spine transpedicular screw insertion (16), we did not observe any neurovascular injury in our small case series. A preoperative CT angiogram of the neck identified the location of the vertebral artery relative to the $\mathrm{C} 7$ pedicle in each of our patients, and we advocate this practice.

The vertebral artery does not uniformly enter the cervical spine. Studies have shown that although the majority of vertebral arteries enter at the C6 transverse foramen (>95\%), a small but considerable portion enter above that (3.6\%) or at C7 $(0.8 \%)(18)$. In addition, $>8 \%$ of patients have a vertebral artery anomaly in the V2 segment (18). Thus, it is paramount to assess the vertebral artery anatomy prior to C7 pedicle screw placement to avoid vascular injury, and we recommend preoperative noninvasive vascular imaging in this clinical scenario.

It is possible that an increased risk of neurovascular injury comes from difficulty visualizing the bony anatomy intraoperatively at the cervicothoracic junction. Due to the orientation of the shoulder, clavicle, and vertebral body, lateral 2D fluoroscopy can be limited in its ability to radiographically visualize anatomic landmarks for screw placement and the location of the placed hardware. Spinal neuronavigation and $3 \mathrm{D}$ intraoperative imaging provide improved guidance for $\mathrm{C} 7$ pedicle screw placement. Post-placement 3D imaging improves the assessment of instrumentation location. Anecdotally, we found C7 pedicle screw placement technically less challenging with spinal neuronavigation as compared to placement with $2 \mathrm{D}$ fluoroscopic guidance. A recent evaluation shows decreased rates of malpositioning of cervicothoracic pedicle screws with spinal neuronavigation (19) as opposed to previously reported rates with freehand techniques (20).

Decompression and stabilization are the main surgical goals in the management of SMD. Cervicothoracic junction and high thoracic tumors can present a challenge to spinal surgeons attempting to stabilize these spinal levels. Our findings add to a small but consequential number of studies showing the effectiveness of $\mathrm{C} 7$ pedicle screws as a superior fixation point in spinal oncology, specifically in metastatic lesions. Spinal surgeons should consider the $\mathrm{C} 7$ pedicle as a fixation point for stabilizing the cervicothoracic junction.

\section{Acknowledgements}

The authors thank Paul H. Dressel BFA for preparation of the illustrations and Elaine C. Mosher MLS for editorial assistance.

Funding: This work was supported by Roswell Park Cancer Institute and National Cancer Institute (NCI) grant P30CA016056.

\section{Footnote}

Conflicts of Interest: Dr. Fabiano receives research support from Arbor Pharmaceuticals. Dr. Thind has no conflicts of interest to declare.

Informed Consent: Written informed consent was obtained from the patients for publication of the images.

\section{References}

1. Deng Z, Xu B, Jin J, et al. Strategies for management of spinal metastases: A comprehensive review. Cancer Transl Med 2015;1:94-100.

2. Laufer I, Rubin DG, Lis E, et al. The NOMS framework: approach to the treatment of spinal metastatic tumors. Oncologist 2013;18:744-51.

3. Johnston TL, Karaikovic EE, Lautenschlager EP, et al. Cervical pedicle screws vs. lateral mass screws: uniplanar fatigue analysis and residual pullout strengths. Spine J 2006;6:667-72.

4. Jones EL, Heller JG, Silcox DH, et al. Cervical pedicle screws versus lateral mass screws. Anatomic feasibility and biomechanical comparison. Spine (Phila Pa 1976) 1997;22:977-82.

5. Barrey C, Cotton F, Jund J, et al. Transpedicular screwing of the seventh cervical vertebra: anatomical considerations and surgical technique. Surg Radiol Anat 2003;25:354-60.

6. Onibokun A, Khoo LT, Bistazzoni S, et al. Anatomical considerations for cervical pedicle screw insertion: the use of multiplanar computerized tomography measurements in 122 consecutive clinical cases. Spine J 2009;9:729-34. 
7. Bach F, Larsen BH, Rohde K, et al. Metastatic spinal cord compression. Occurrence, symptoms, clinical presentations and prognosis in 398 patients with spinal cord compression. Acta Neurochir (Wien) 1990;107:37-43.

8. Fanous AA, Fabiano AJ. Surgical management of spinal metastatic disease. J Neurosurg Sci 2017;61:316-24.

9. Tokuhashi Y, Ajiro Y, Umezawa N. Outcome of treatment for spinal metastases using scoring system for preoperative evaluation of prognosis. Spine (Phila Pa 1976) 2009;34:69-73.

10. Rao S, Badani KM, Jamieson K, et al. Pitfalls in the surgical management of cervical spine injuries. Eur Spine J 1996;5:153-60.

11. Placantonakis DG, Laufer I, Wang JC, et al. Posterior stabilization strategies following resection of cervicothoracic junction tumors: review of 90 consecutive cases. J Neurosurg Spine 2008;9:111-9.

12. Oda I, Abumi K, Ito M, et al. Palliative spinal reconstruction using cervical pedicle screws for metastatic lesions of the spine: a retrospective analysis of 32 cases. Spine (Phila Pa 1976) 2006;31:1439-44.

13. Mazel C, Hoffmann E, Antonietti P, et al. Posterior cervicothoracic instrumentation in spine tumors. Spine (Phila Pa 1976) 2004;29:1246-53.

14. Hong JT, Tomoyuki T, Udayakumar R, et al. Biomechanical comparison of three different types of $\mathrm{C} 7$

Cite this article as: Thind $\mathrm{H}$, Fabiano AJ. The $\mathrm{C} 7$ pedicle as a superior fixation point in spinal stabilization for spinal metastatic disease. J Spine Surg 2018;4(1):156-161. doi: 10.21037/ jss.2018.03.07 fixation techniques. Spine (Phila Pa 1976) 2011;36:393-8.

15. Rath SA, Moszko S, Schaffner PM, et al. Accuracy of pedicle screw insertion in the cervical spine for internal fixation using frameless stereotactic guidance. J Neurosurg Spine 2008;8:237-45.

16. Abumi K, Shono Y, Ito M, et al. Complications of pedicle screw fixation in reconstructive surgery of the cervical spine. Spine (Phila Pa 1976) 2000;25:962-9.

17. Le H, Balabhadra R, Park J, et al. Surgical treatment of tumors involving the cervicothoracic junction. Neurosurg Focus 2003;15:E3.

18. Wakao N, Takeuchi M, Kamiya M, et al. Variance of cervical vertebral artery measured by CT angiography and its influence on C7 pedicle anatomy. Spine (Phila Pa 1976) 2014;39:228-32.

19. Barsa P, Frohlich R, Sercl M, et al. The intraoperative portable CT scanner-based spinal navigation: a viable option for instrumentation in the region of cervicothoracic junction. Eur Spine J 2016;25:1643-50.

20. Hojo Y, Ito M, Suda K, et al. A multicenter study on accuracy and complications of freehand placement of cervical pedicle screws under lateral fluoroscopy in different pathological conditions: CT-based evaluation of more than 1,000 screws. Eur Spine J 2014;23:2166-74. 